\title{
Video Foreground Detection Based on Symmetric Alpha-Stable Mixture Models
}

\author{
Harish Bhaskar, Lyudmila Mihaylova, Senior Member, IEEE, and Alin Achim, Senior Member, IEEE
}

\begin{abstract}
Background subtraction (BS) is an efficient technique for detecting moving objects in video sequences. A simple BS process involves building a model of the background and extracting regions of the foreground (moving objects) with the assumptions that the camera remains stationary and there exist no movements in the background. These assumptions restrict the applicability of BS methods to real-time object detection in video. In this paper, we propose an extended cluster BS technique with a mixture of symmetric alpha stable $(S \alpha S)$ distributions. An on-line self-adaptive mechanism is presented that allows automated estimation of the model parameters using the log moment method. Results over real video sequences from indoor and outdoor environments, with data from static and moving video cameras are presented. The $S \alpha S$ mixture model is shown to improve the detection performance compared with a cluster BS method using a Gaussian mixture model and the method of Li et al. [11].
\end{abstract}

Index Terms-automatic object detection, background subtraction, segmentation, alpha stable distribution

\section{INTRODUCTION}

Moving object detection in video sequences represents a critical component of many modern video processing systems. The standard approach to object detection is Background Subtraction (BS), that attempts to build a representation of the background and detect moving objects by comparing each new frame with this representation [4]. A number of different BS techniques have been proposed in the literature and some of the popular methods include mixture of Gaussians [24], kernel density estimation [6], colour and gradient cues [9], high level region analysis [22], hidden Markov models [21], and Markov random fields [14]. Basic BS techniques detect foreground objects as the difference between two consecutive video frames, operate at pixel level and are applicable to static backgrounds [4]. Although the generic BS method is simple to understand and implement, the disadvantages of the frame difference $\mathrm{BS}$ is that it does not provide a mechanism for choosing the parameters, such as the detection threshold, and it is unable to cope with multi-modal distributions. One of the important techniques able to cope with multi-modal

Harish Bhaskar is with Khalifa University of Science Technology and Research, UAE, email: harish.bhaskar@kustar.ac.ae. Lyudmila Mihaylova is with Lancaster University, UK, Email: mila.mihaylova@lancaster.ac.uk. Alin Achim is with the University of Bristol, UK, Email: alin.achim@bristol.ac.uk. We acknowledge the support of the UK MOD Data and Information Fusion DTC (Tracking Cluster project DIFDTC/CSIPC1/02) and EU COST action TU0702. Corresponding author: L. Mihaylova, mila.mihaylova@lancaster.ac.uk. Manuscript received May 2008, revised November, 2009 and March 2010. Copyright (c) 2010 IEEE. Personal use of this material is permitted. However, permission to use this material for any other purposes must be obtained from the IEEE by sending an email to pubs-permissions@ieee.org. background distributions and to update the detection threshold makes use of Gaussian mixture models (GMMs). The model proposed in [24] describes each pixel as a mixture of Gaussians and an on-line update of this model. The larger Gaussian components correspond to the background and this is used to generate the background model. An algorithm for background modeling and BS based on Cauchy statistical distribution [13] is shown to be robust and adaptive to dynamic changes of the background scene and more cost effective than the GMM as it does not involve any exponential operation.

In [11] the foreground objects are detected in complex environments. The background appearance is characterised by principal features (spectral, spatial and temporal) and their statistics, at each pixel. However, the learning method in [11] requires 'training' since it relies on look up tables for the features and adapts them to the changes of environment. The CBS- $S \alpha S$ technique that we propose does not need such look up tables for the image features and is a cluster-based technique, which makes it different from [11]. According to our knowledge only one recent work [18] considers mixtures of $S \alpha S$ distributions for off-line data analysis and does not seem suitable for real-time object detection.

In this paper, we propose a novel cluster BS (CBS) technique based on $S \alpha S$ distributions, which technique we call CBS-S $\alpha S$. The main contributions of the paper are threefold. Firstly, the BS process is performed at cluster level as opposed to pixel level methods that are commonly used [6], [24], [4]. The CBS- $S \alpha S$ method reduces significantly the clutter noise that arises due to slight variations in the pixel intensities within regions belonging to the same object. Secondly, due to their heavy tails, $S \alpha S$ distributions can help handling dynamic changes in a scene, and hence they model moving backgrounds and moving camera in a better way than the GMM. Results of modeling the background of a moving image sequence can be best obtained while operating with estimated values of the characteristic exponent parameter of the $S \alpha S$ distribution, rather than with fixed values corresponding to the Gaussian or Cauchy case. By estimating the parameters of the $\alpha$ stable distribution, the probability density function (PDF) of clusters of pixels can be faithfully represented and a reliable model of the background can be obtained. Thirdly, we show that a mixture of $S \alpha S$ distributions can represent well the multimodality and guarantees reliable object detection. A wide range of tests is performed on indoor and outdoor environment, on data from a static and moving cameras.

The remaining part of the paper is organised as follows. Section II presents the proposed CBS- $S \alpha S$ technique. A comparison of the CBS- $S \alpha S$ with a CBS-GMM BS and 
the background appearance method of $\mathrm{Li}$ et al. [11] on real video sequences is presented in Section III. Conclusions are summarised in Section IV.

\section{The Proposed CBS- $S \alpha S$ Technique}

In contrast with conventional BS techniques such as [6], [24], [4] that operate at pixel level, the developed BS technique makes use of the advantages of the cluster image representation. Here, an image frame at time instant $k$ is subdivided into constituent clusters $c_{k}^{i}$, $(0 \leq i \leq q)$, where $q$ is the total number of clusters present in the image. Automatic clustering is performed with vector quantisation and using colour features [15].

The problem of CBS involves a decision on whether a cluster $\boldsymbol{c}_{k}^{i}$ belongs to the background ( $b G$ ) or foreground $(f G)$ object from the ratio of PDFs:

$$
\frac{p\left(b G \mid \boldsymbol{c}_{k}^{i}\right)}{p\left(f G \mid \boldsymbol{c}_{k}^{i}\right)}=\frac{p\left(\boldsymbol{c}_{k}^{i} \mid b G\right) p(b G)}{p\left(\boldsymbol{c}_{k}^{i} \mid f G\right) p(f G)}
$$

where, the vector $\boldsymbol{c}_{k}^{i}=\left(c_{1, k}^{i}, \ldots, c_{\ell, k}^{i}\right)$ characterises the $i$-th cluster $(0 \leq i \leq q)$ at time instant $k$, containing $\ell$ number of pixels such that $[\mathrm{Im}]_{k}=\left[\boldsymbol{c}_{k}^{1}, \ldots, \boldsymbol{c}_{k}^{q}\right]$ is the whole image; $p\left(b G \mid c_{k}^{i}\right)$ is the PDF of the $b G$, evaluated using a certain feature (e.g. colour or edges) of the cluster $\boldsymbol{c}_{k}^{i} ; p\left(f G \mid \boldsymbol{c}_{k}^{i}\right)$ is the PDF of the $f G$ of the same cluster $c_{k}^{i} ; p\left(c_{k}^{i} \mid b G\right)$ refers to the PDF of the cluster feature given a model for the $b G$ and $p\left(\boldsymbol{c}_{k}^{i} \mid f G\right)$ is the appearance model of the $f G$ object. In our cluster BS technique the decision that a cluster belongs to a $b G$ is made if:

$$
p\left(\boldsymbol{c}_{k}^{i} \mid b G\right)>\text { threshold }\left(=\frac{p\left(\boldsymbol{c}_{k}^{i} \mid f G\right) p(f G)}{p(b G)}\right) .
$$

The appearance of the $f G$, characterised by the PDF $p\left(\boldsymbol{c}_{k}^{i} \mid f G\right)$, is assumed uniform. The $b G$ model represented as $p\left(\boldsymbol{c}_{k}^{i} \mid b G\right)$ is estimated from a training set $\Re_{k}=\left\{\boldsymbol{c}_{k}^{i}, \ldots, \boldsymbol{c}_{k-T}^{i}\right\}$ which is a rolling collection of images over a specific update time $T$. The time $T$ is crucial since its update determines the model ability to adapt to illumination changes and to handle appearances and disappearances of objects in a scene. If the frame rate is known, the time period $T$ can be adapted, e.g., as a ratio $T=\frac{N}{f p s}$ between the number $N$ of frames obtained through the online process and the frame rate, fps, frames per second. Since the threshold is a scalar, the decision in (2) is made from the average of the distributions of all pixels within the cluster $\boldsymbol{c}_{k}^{i}$.

\section{A. Alpha Stable Distributions}

The appeal of $S \alpha S$ distributions as a statistical model for signals derives from some important theoretical and empirical reasons [19]. Generally, there is no closed-form expression for the PDF of $S \alpha S$ distributions. A convenient way of defining them is by their characteristic function $\varphi(\boldsymbol{c})=\exp (j \delta \boldsymbol{c}-$ $\gamma|c|^{\alpha}$ ), where $\alpha$ is the characteristic exponent parameter, with values $0<\alpha \leq 2$ that controls the heaviness of the tails of the density function, $\delta$ is the location parameter $(-\infty<\delta<\infty)$ that corresponds to the mean for $1<\alpha \leq 2$, and to the median for $0<\alpha \leq 1$ and $\gamma$ is the dispersion parameter $(\gamma>0)$, which determines the spread of the density around the location parameter. A $S \alpha S$ distribution, characterised by the above three parameters is denoted by $\mathcal{S}(\alpha, \gamma, \delta)$. In fact, no closedform expressions for the general symmetric $\alpha$ stable PDF exist, except for the Gaussian and Cauchy members. Specifically, the case $\alpha=2$ corresponds to the Gaussian distribution and the PDF has the form

$$
f_{\alpha=2}(\gamma, \delta ; \boldsymbol{c})=\frac{1}{\sqrt{4 \pi \gamma}} \exp \left\{-\frac{(\boldsymbol{c}-\delta)^{2}}{4 \gamma}\right\} .
$$

The case $\alpha=1$ corresponds to the Cauchy distribution, for which the PDF is given by

$$
f_{\alpha=1}(\gamma, \delta ; \boldsymbol{c})=\frac{\gamma}{\pi\left[\gamma^{2}+(\boldsymbol{c}-\delta)^{2}\right]} .
$$

\section{B. SaS Mixture Models}

The PDFs $\widetilde{p}\left(c_{k}^{i} \mid \Re_{k}, b G+f G\right)$ of the $f G$ and $b G$ can be calculated as a mixture

$$
\widetilde{p}\left(\boldsymbol{c}_{k}^{i} \mid \Re_{k}, b G+f G\right)=\sum_{m=1}^{M} \widetilde{\pi}_{m, k} \varphi\left(\boldsymbol{c}_{k}^{i} ; \widetilde{\boldsymbol{\alpha}}_{k}, \widetilde{\gamma}_{m, k}\right),
$$

of an $M$ component $S \alpha S$ PDFs $\varphi\left(\boldsymbol{c}_{k}^{i} ; \widetilde{\boldsymbol{\alpha}}_{k}, \widetilde{\gamma}_{m, k}\right)$ (with parameters $\widetilde{\boldsymbol{\alpha}}_{k}$, the characteristic exponent and $\widetilde{\gamma}_{m, k}$, dispersion parameter). The weighting coefficients $\widetilde{\pi}_{m, k}$ are calculated as shown in the next subsection.

\section{Iterative Log-Moment Estimation}

The most important parameters of a $S \alpha S$ distribution are the characteristic exponent $\alpha$ and dispersion parameter $\gamma$. The location parameter $\delta$ is often assumed to be zero, i.e., the measurements are normalised with respect to the origin. Several methods for estimating these parameters have been introduced in [12]. In our CBS-S $\alpha S$ technique the parameters of the $S \alpha S$ distribution are evaluated based on the log-moment estimation method. The update of the parameter estimates $\tilde{\boldsymbol{\alpha}}_{(1, k)}, \tilde{\boldsymbol{\alpha}}_{(2, k)}, \ldots, \tilde{\boldsymbol{\alpha}}_{(M, k)}$ and $\tilde{\boldsymbol{\gamma}}_{(1, k)}$, $\tilde{\gamma}_{(2, k)}, \ldots, \tilde{\gamma}_{(M, k)}$ at time instant $k$ is performed, respectively, from the estimates $\tilde{\boldsymbol{\alpha}}_{(1, k-1)}, \tilde{\boldsymbol{\alpha}}_{(2, k-1)}, \ldots, \tilde{\boldsymbol{\alpha}}_{(M, k-1)}$ and $\tilde{\gamma}_{(1, k-1)}, \tilde{\gamma}_{(2, k-1)}, \ldots, \tilde{\gamma}_{(M, k-1)}$ at a previous time $k-1$. According to the log moment estimation technique, if $\Re_{k}$ is a real $S \alpha S$ random variable, then its $p$-th order moment satisfies the relation $E\left(\left|\Re_{k}\right|^{p}\right)=E\left(e^{p l o g\left|\Re_{k}\right|}\right)=E\left(e^{p V}\right)$, where $-1<p<\alpha$ and $V=\log \left|\Re_{k}\right|$ corresponds to a $\log |S \alpha S|$ process with $\boldsymbol{\mu}_{k}$ and $\sigma_{k}$ representing the mean and variance of the $\Re_{k}$ samples. The estimates of the mean and variances, $\widetilde{\boldsymbol{\mu}}_{1, k}, \ldots, \widetilde{\boldsymbol{\mu}}_{M, k}$ and $\widetilde{\sigma}_{1, k}^{2}, \ldots, \widetilde{\sigma}_{M, k}^{2}$, respectively of the $\Re_{k}$ samples can be represented as in [23] with

$$
\begin{gathered}
\widetilde{w}_{m, k+1}=\widetilde{w}_{m, k}+\frac{1}{T_{k}}\left(o_{m, k}-\widetilde{w}_{m, k}\right), \\
\widetilde{\boldsymbol{\mu}}_{m, k+1}=\widetilde{\boldsymbol{\mu}}_{m, k}+o_{m, k}\left(\frac{1}{T_{k} \widetilde{w}_{m, k}}\right) \boldsymbol{\delta}_{m, k}, \\
\widetilde{\sigma}_{m, k+1}^{2}=\widetilde{\sigma}_{m, k}^{2}+o_{m, k}\left(\frac{1}{T_{k} \widetilde{w}_{m, k}}\right)\left(\boldsymbol{\delta}_{m, k}^{\prime} \boldsymbol{\delta}_{m, k}-\sigma_{m, k}^{2}\right),
\end{gathered}
$$

where $\boldsymbol{\delta}_{m, k}=\boldsymbol{c}_{k}^{i}-\widetilde{\boldsymbol{\mu}}_{m, k},{ }^{\prime}$ denotes the transpose operation, $o_{m, k}$ refers to the ownership of the new cluster and defines the closeness of this cluster to a particular $S \alpha S$ component, 
and $m=1, \ldots, M$. The dispersion parameter $\gamma_{m, k}$ at any time instant $k$ can be updated iteratively using the following equations [12]

$$
\begin{array}{r}
\frac{\log \gamma_{m, k}}{\boldsymbol{\alpha}_{m, k}}=\frac{k-1}{k} \\
\left(\frac{C_{e}\left(1-\boldsymbol{\alpha}_{m, k-1}\right)+\log \gamma_{m, k-1}}{\boldsymbol{\alpha}_{m, k-1}}\right) \\
+\frac{\boldsymbol{\mu}_{m, k}}{k}+C_{e}\left(1-\frac{1}{\boldsymbol{\alpha}_{m, k}}\right)
\end{array}
$$

and similarly the characteristic exponent $\alpha_{k}$ at time instant $k$ can be updated as follows [12]

$$
\begin{gathered}
\frac{\pi^{2}}{6}\left(\frac{1}{\boldsymbol{\alpha}_{m, k}^{2}}+\frac{1}{2}\right)=\frac{k-1}{k^{2}}\left(\frac{C_{e}\left(1-\boldsymbol{\alpha}_{m, k-1}\right)+\log \gamma_{m, k-1}}{\boldsymbol{\alpha}_{m, k-1}}-\boldsymbol{\mu}_{m, k}\right)^{2} \\
+\frac{\pi^{2}(k-1)}{6 k}\left(\frac{1}{\boldsymbol{\alpha}_{m, k-1}^{2}}+\frac{1}{2}\right)+\frac{1}{k} \sigma_{m, k}
\end{gathered}
$$

where $C_{e}=0.57721566 \ldots$ is the Euler constant. The accuracy of this parameter estimation technique increases with the increase in sample size. The ownership of any new cluster is set to 1 for "close" components and the others are set to zero. A cluster is close to a component iff the Mahalanobis distance between the component and the cluster centre is, e.g., less than 3. If there are no "close" components, a component is generated with $\widetilde{w}_{m+1, k}=1 / T_{k}$, with an initial mean $\widetilde{\boldsymbol{\mu}}_{0}$ and variance $\widetilde{\sigma}_{0}^{2}$. The model presents clustering of components and the background is approximated with the $B$ largest components, $\widetilde{p}\left(\boldsymbol{c}_{k}^{i} \mid \Re_{k}, b G\right) \sim \sum_{m=1}^{B} \widetilde{w}_{m, k} \varphi\left(\boldsymbol{\alpha}_{m, k}, \boldsymbol{\gamma}_{m, k}\right)$, $B=\operatorname{argmin}_{b}\left(\sum_{m=1}^{b} \widetilde{w}_{m, k}>\left(1-c_{f}\right)\right)$, where $b$ is a variable defining the number of clusters considered and $c_{f}$ is the proportion of the data that belong to $f G$ objects without influencing the background model. The proportion between the pixels from the $f G$ and the pixels from the $b G$ is assumed constant in most models [24]. This assumption is not valid when videos of objects are captured from a close proximity. In such circumstances, the proportion of pixels belonging to the objects of interest, i.e., the $f G$ pixels, are much higher than the $b G$ pixels. The ratio defining the percentage of $f G$ and $b G$ pixels can be updated from the training set as follows:

$$
c_{f}=\frac{\widetilde{p}\left(c_{k}^{i} \mid \Re_{k}, f G\right)}{\widetilde{p}\left(c_{k}^{i} \mid \Re_{k}, b G\right)} .
$$

\section{Results AND ANALysis}

The performance of the proposed CBS- $S \alpha S$ technique has been validated over a real video sequences CAVIAR [1], PETS [2], data taken with static and moving video cameras, from outdoor and indoor scenarios [16]. The CBS- $S \alpha S$ technique is compared with the CBS-GMM [3] and with the technique for complex $b G$ and $f G$ object detection proposed in [11]. In our experiments (except where is it explicitly stated), we have chosen the number of mixture components for the CBS- $S \alpha S$ and CBS-GMM to be 3 .

\section{A. Static Camera Video Sequence}

We first compare the performance of the proposed CBS-S $\alpha S$ technique with the CBS-GMM [3] performance using real video sequences taken with a stationary camera. In general, both the CBS-GMM and the CBS-S $\alpha S$ models perform well. It can be observed from the results on Figure 1 that the
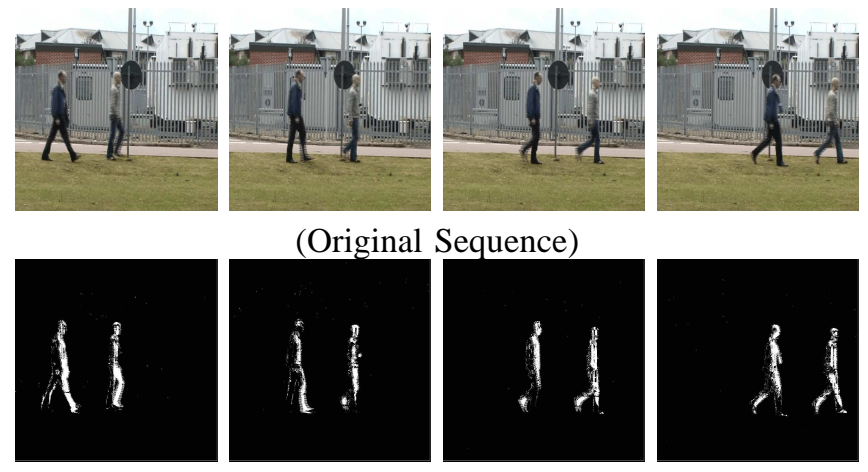

(a: Results from the CBS-GMM)
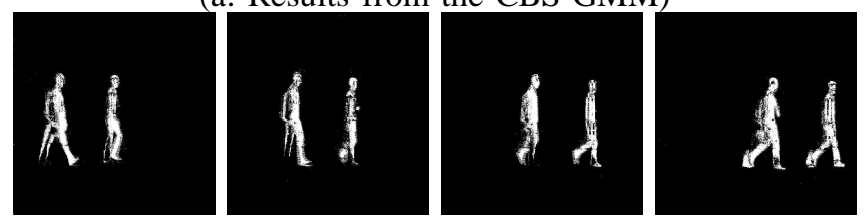

(b: Results from the CBS- $S \alpha S$ )

Fig. 1. Results from: (a) CBS-GMM [3], (b) proposed CBS-S $\alpha S$ model on sequence 1 (static camera), DIF DTC testing data [10].

proposed mixture of $S \alpha S$ distributions is able to avoid oversegmentation, to cope well with the multi-modality and hence to represent in a better way the object of interest than the GMM. In the next subsection, results on video data captured with a moving camera and movements in the $b G$ are presented.

\section{B. Moving Camera Video Sequence}

Deep sea water video sequences are particularly challenging due to the video camera movement. The object of interest is
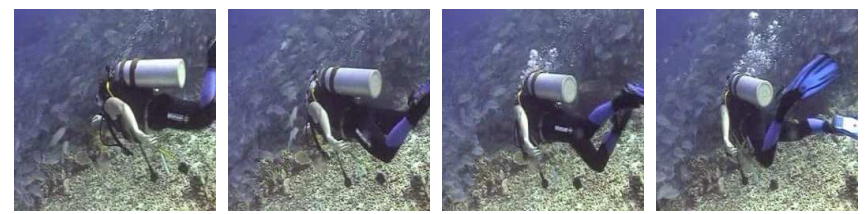

(Original Sequence)
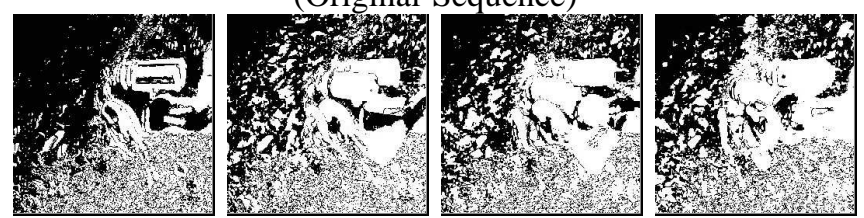

(a: Results from the CBS-GMM)
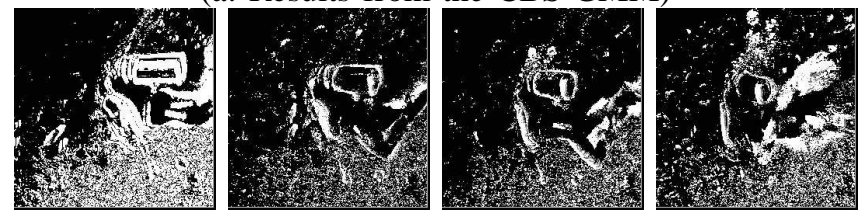

(b: Results from the CBS- $S \alpha S$ )

Fig. 2. Results from the (a) CBS-GMM [3] and (b) proposed CBS-S $\alpha S$ model on sequence 2 (moving camera sequence)

a deep sea diver who is also in motion. There are sources of multi-modality, moving $b G$ and the camera is moving. From Fig. 2 a) and b) is evident that the $S \alpha S$ CBS reduces significantly the level of clutter. We performed additional tests on another video sequence, corresponding to an outdoor scenario (taken from [16]). The results from Fig. 3 show that the 

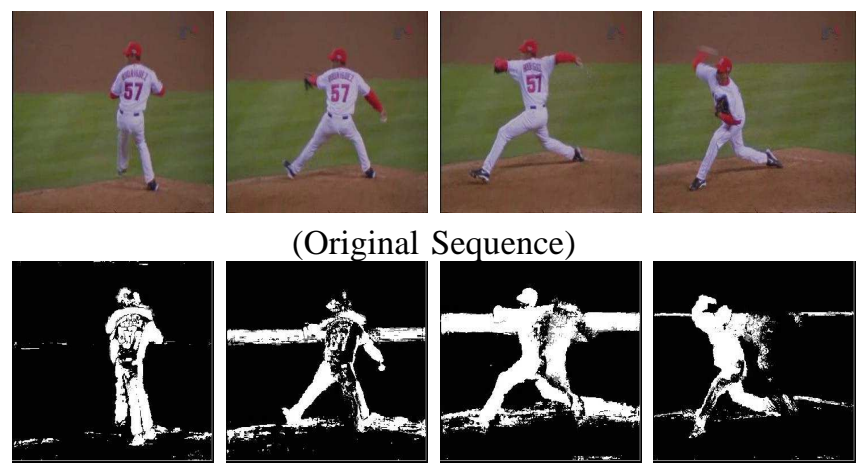

(Original Sequence)
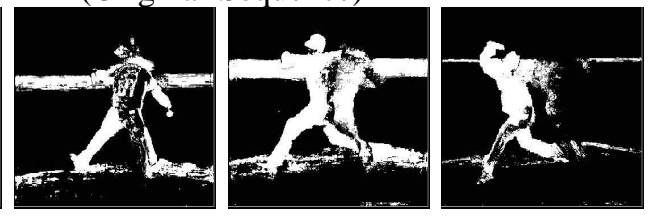

(a: Results from the CBS-GMM)
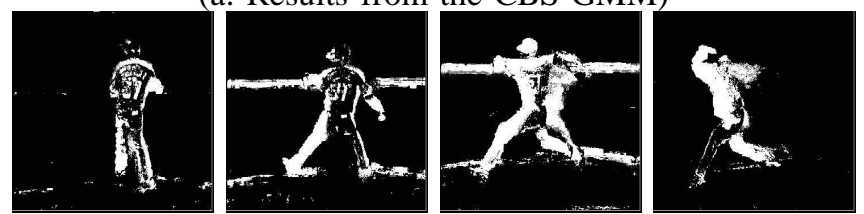

(b: Results from the CBS- $S \alpha S$ )

Fig. 3. Results from: (a) CBS-GMM [3], (b) proposed CBS- $S \alpha S$ model on sequence taken with moving camera (public data [16]).

proposed CBS- $S \alpha S$ technique reduces the clutter noise and to cope with camera displacements and small movements in the $b G$. The $S \alpha S$ densities with heavy tails can accommodate the variations in the $b G$ and possesses higher robustness to illumination changes than the CBS-GMM.

\section{Quantitative Analysis}

Recall and precision quantify how well an algorithm matches the ground truth. Recall [5] is calculated as the ratio of the number of $f G$ pixels correctly identified to the number of $f G$ pixels in the ground truth and precision is computed as a ratio of the number of $f G$ pixels correctly identified to the number of $f G$ pixels detected. In Figure 4 it can be observed
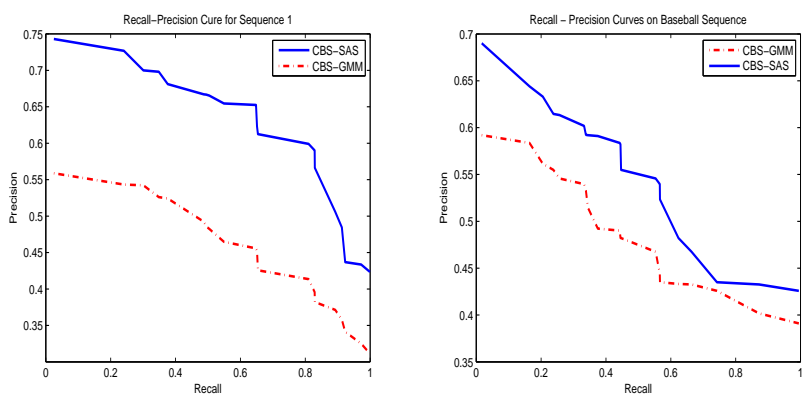

Fig. 4. Recall-Precision curves of the CBS-GMM [3] and proposed CBS$S \alpha S$ model, for sequences 2 and 3.

that both techniques show a steady decrease in precision with increase in recall. However, the proposed CBS- $S \alpha S$ algorithm (blue line) displays higher levels of precision for the same values of the recall than the CBS-GMM (red dashed line). The higher the rate of precision implies a greater rate of correct classification of $f G$ pixels [5].

The performance of the CBS-S $\alpha S$ technique was further tested over a large sequences from CAVIAR [1] and PETS [2] datasets. In addition to the recall and precision measures, the ratio $S(A, B)=\{A \cap B\} /\{A \cup B\}$ [7] between the intersection of the ground truth and segmented regions over the union of the ground truth and segmented regions is used to evaluate the performance of the CBS-S $\alpha S$, CBS-GMM algorithms and the algorithm of $\mathrm{Li}$ et al. [11]. The accuracy of the segmentation process increases with increasing the values of $S$. If $S>0.5$ the performance of segmentation is generally considered good and nearly perfect for values of $S>0.8$. The average values of precision, recall and $S$-ratio are shown in Table I calculated on CAVIAR data [1], from indoor environment and with the camera above the level of the walking person. According to the these results the CBS-

Table 1: Precision, recall and $S$-ratio with CAVIAR data [1]

\begin{tabular}{|c|c|c|c|c|c|c|c|c|c|c|}
\hline \multirow{3}{*}{$\begin{array}{l}\text { Sequence } \\
\text { Number }\end{array}$} & \multirow{3}{*}{$\begin{array}{l}\text { CAVIAR } \\
\text { Video }\end{array}$} & \multicolumn{3}{|c|}{ Recall } & \multicolumn{3}{|c|}{ Precision } & \multicolumn{3}{|c|}{ S-Ratio } \\
\hline & & CBS & CBS & & CBS & CBS & & CBS & CBS & \\
\hline & & GMM & SAS & li & GMM & SAS & Li & GMM & SAS & Li \\
\hline 1 & Browse 1 & 0.42 & 0.48 & 0.46 & 0,31 & 0.47 & 0,39 & 0.35 & 0.57 & 0.51 \\
\hline 2 & Browse2 & 0.31 & 0.54 & 0.49 & 0.38 & 0.52 & 0.44 & 0.23 & 0.48 & 0.39 \\
\hline 3 & Walk1 & 0.23 & 0.47 & 0.41 & 0.41 & 0.48 & 0.42 & 0.33 & 0.61 & 0.52 \\
\hline 4 & Walk2 & 0.26 & 0.38 & 0.34 & 0.37 & 0.61 & 0.51 & 0.28 & 0.59 & 0.50 \\
\hline 5 & Fight Chase & 0.32 & 0.61 & 0.53 & 0.41 & 0.53 & 0.27 & 0.31 & 0.67 & 0.48 \\
\hline
\end{tabular}

$S \alpha S$ technique achieves the highest values for the precision, recall, and $S$-ratio followed by the algorithm of $\mathrm{Li}$ et al. [11] and then by the CBS-GMM algorithm. In order to explain this performance of the CBS-S $\alpha S$ technique, we consider the estimates of $\alpha$ (the characteristic exponent) over different frames of a video sequence (plotted in blue) taken with a static camera and a video sequence (plotted in red) taken with a moving camera from the PETS 2001 dataset. Figure 6 (a) shows that the value of $\alpha$ for the static camera is around 1.5 to 2 . For moving camera the value ranges between 1 and 2 (nearly 1 means that the distribution is nearly Cauchy).

The better performance of the CBS-S $\alpha S$ with respect to the CBS-GMM technique can be understood when we consider the grey level intensity representing the $b G$ subject to illumination changes over a short period of time. For the PETS sequence the histograms are given in Figure 7. The heavy tailed multimodal histogram cannot be represented well by the standard GMM as seen from Figure 7. Sample distributions of the GMM are compared with the $S \alpha S$ model of the intensity. Clearly, the mixture of $S \alpha S$ distributions (dashed dot lines) is models variations in intensity in a better way than the standard GMM. Similar experiments with slight variations of the $b G$ due to camera movements have established the superiority of the CBS-S $\alpha S$ model.

\section{CONClusions AND Future Work}

A novel approach for automatic object detection based on cluster BS with $S \alpha S$ distribution was introduced. The heavy tail $S \alpha S$ distributions allow to cope with slight movements in the background, camera shakes and clutter noise. An adaptive framework for parameter estimation is proposed that allows the model to adapt to environmental changes. A comparison of the model to its counterpart CBS-GMM model [3] is presented. Experimental results show that the CBS- $S \alpha S$ algorithm has efficient performance measured by precision, recall and $S$ ratios and outperforms both the CBS algorithms with a GMM 

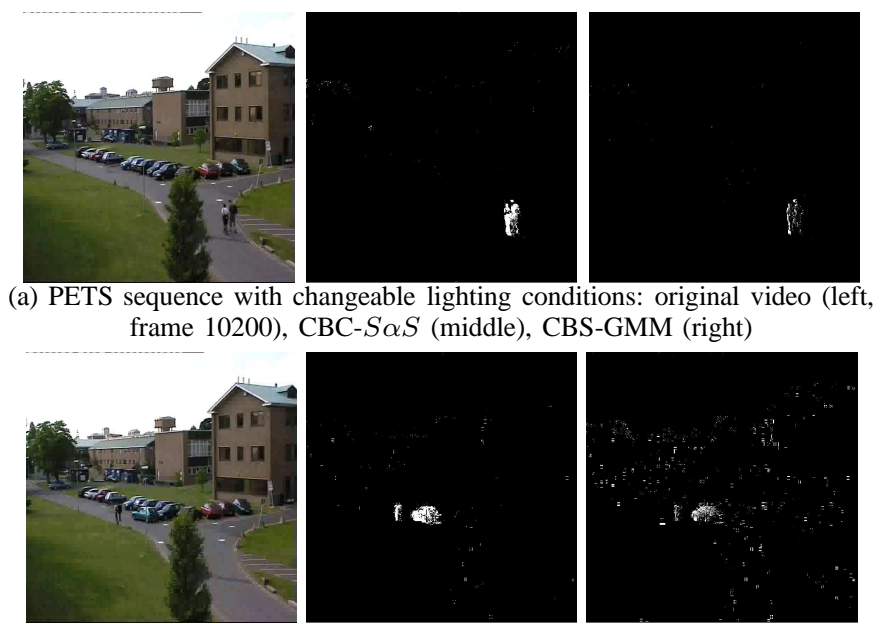

(b) PETS sequence with changeable lighting conditions: original video (left, frame 11044), CBC-S $\alpha S$ (middle), CBS-GMM (right)
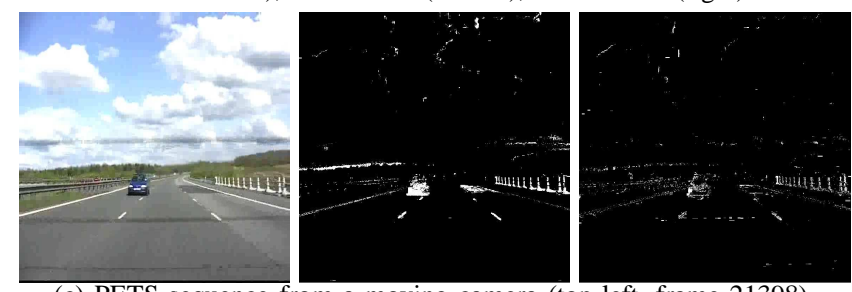

(c) PETS sequence from a moving camera (top left, frame 21398), CBC- $S \alpha S$ (middle), CBS-GMM (right)

Fig. 5. Results on PETS sequences [2]

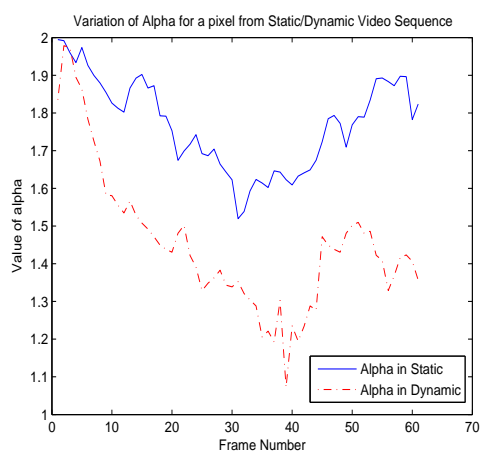

Fig. 6. (a) Estimated $\alpha$ : in video from a static camera ( $\alpha$ in static) and from a moving camera ( $\alpha$ in dynamic)

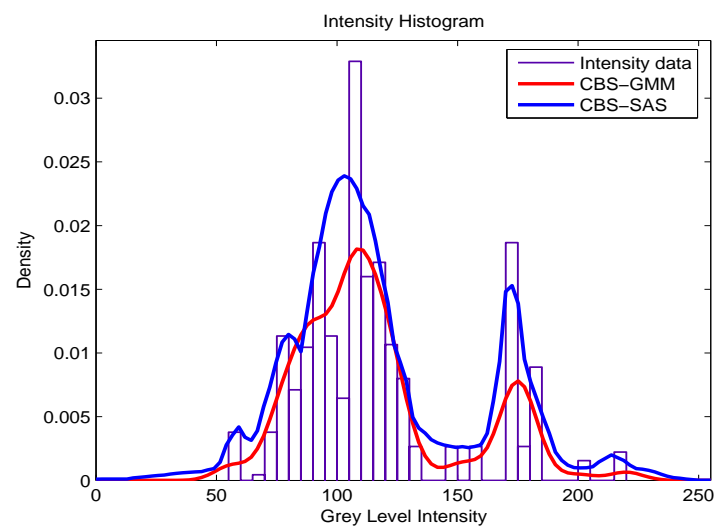

Fig. 7. Intensity histogram of a background pixel: CBS-S $\alpha S$ and CBS-GMM

and the algorithm of $\mathrm{Li}$ et al. [11]. The model has relatively low memory requirements and can process at the rate of $15-20$ fps on a Intel Duo Core processor machine. Our future work will be focussed on BS for videos from moving cameras, with scenarios similar to the considered in [20], [17], [8].

\section{REFERENCES}

[1] CAVIAR, http://homepages.inf.ed.ac.uk/rbf/caviardata1/, 2003.

[2] PETS: Performance evaluation of tracking and surveillance, available at http://www.cvg.rdg.ac.uk/slides/pets.html, 2006.

[3] H. Bhaskar, L. Mihaylova, and S. Maskell. Automatic target detection based on background modeling using adaptive cluster density estimation. In $L N C S$, pages 130-134, 2007.

[4] R. Cucchiara, C. Grana, M. Piccardi, and A. Prati. Detecting moving objects, ghosts and shadows in video streams. IEEE Trans. PAMI, 25(10):1337-1342, 2005.

[5] J. Davis and M. Goadrich. The relationship between precision-recall and ROC curves. In Proc. ICML, pages 233-240, 2006.

[6] A. Elgammal, D. Harwood, and L. Davis. Non-parametric model for background subtraction. In Proc. 6th European Conf. on Computer Vision, pages II: 751-767, June/July 2000.

[7] G. Gerig, M. Jomier, and M. Chakos. Valmet: A new validation tool for assessing and improving 3D object segmentation. In Proceedings of MICCAI, LNCS 2208, pages 516-523, 2001.

[8] E. Hayman and J.-O. Eklundh. Statistical background subtraction for a mobile observer. In Proceedings of the 9th International Conf. on Computer Vision, pages 67-74, 2003.

[9] O. Javed, K. Shafi que, and M. Shah. A hierarchical approach to robust background subtraction using color and gradient information. In Proc. MOTION'02, pages 22-27, 2002.

[10] J.J. Lewis, S.G. Nikolov, A. Loza, E. Fernandez-Canga, N. Cvejic, L. Li, A. Cardinali, C.N. Canagarajah, D.R. Bull, T. Riley, D. Hickman, and M.I. Smith. The Eden project multi-sensor data set. Technical report, Univ. of Bristol, UK, 2006.

[11] L. Li, W. Huang, I. Yu-Hua Gu, and Q. Tian. Statistical modeling of complex backgrounds for foreground object detection. IEEE Trans. on Image Processing, 13(11):1459-1472, 2004.

[12] X. Ma and C. Nikias. Parameter estimation and blind channel identifi cation in impulsive signal environment. IEEE Tran. Sign. Proc., 43(12):2884-2897, Dec. 1995.

[13] Y. Ming, J. Jiang, and J. Ming. Background modeling and subtraction using a local-linear-dependence-based Cauchy statistical model. In Proc. 7th International Conf. on Digital Image Computing: Techniques and Applications, pages 469-478, 2003.

[14] N.Paragios and V.Ramesh. A MRF-based real-time approach for subway monitoring. In Proc. CVPR, pages 1034-1040, 2001.

[15] A. Payne, H. Bhaskar, and L. Mihaylova. Multi-resolution learning vector quantisation based automatic colour clustering. In Proc. 11th Conf. on Inform. Fusion, pages 1934-1939, 2008.

[16] D. Ramanan, D. Forsyth, and A. Zisserman. Tracking people by learning their appearance. IEEE Tr. PAMI, 29(1):65-81, 2007.

[17] Y. Ren, C.-S. Chua, and Y.-K. Ho. Statistical background modeling for non-stationary camera. Pattern Recogition Letters, 24(1-3):183-196, 2003.

[18] D. Salas-Gonzaleza, E. E. Kuruoglu, and D. P. Ruiz. Modelling with mixture of symmetric stable distributions using Gibbs sampling. Signal Processing, 90(3):774-783, 2010.

[19] M. Shao and C. Nikias. Signal processing with fractional lower order moments: Stable processes and their applications. Proceedings of the IEEE, 81(7):986 - 1010, July 1993.

[20] Y. Sheikh, O. Javed, and T. Kanade. Background subtraction for freely moving cameras. In Proc. of IEEE ICCV. 2009.

[21] B. Stenger, V. Ramesh, N. Paragios, F. Coetzec, and J.M. Buhmann. Topology free hidden Markov models: application to background modeling. In Proc. of the International Conf. on Computer Vision, pages 294-301, 2001.

[22] K. Toyama, J. Krumm, B. Brumitt, and B. Meyers. Wallflower: Principles and practice of background maintenance. In Proc. Intl. Conf. Comp. Vision, pages 255-261, 1999.

[23] Z. Zivkovic. Improved adaptive Gausian mixture model for background subtraction. In Proc. ICPR, volume 28 - 31, 2004.

[24] Z. Zivkovic and F. van der Heijden. Effi cient adaptive density estimation per image pixel for the task of background subtraction. Pattern Recognition Letters, 27(7):773-780, 2006. 